DOI: http://dx.doi.org/10.21123/bsj.2016.13.1.0001 دراسة مجتمع لافقريات التربة في احد بساتين نخيل التمر في بغداد / العراق

صبا سالم سلمان البياتي

*يفاء جواد جوير

\author{
*قمس علوم الحياة / كلبة العلوم للبنات / جامعة بغداد

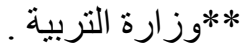

البريد الاكتروني: hjaweir@yahoo.com

استلام البحث 23، تشرين الثاني، 2014

قبول النشر 11، كانون الثاني، 2315

(c) () ()

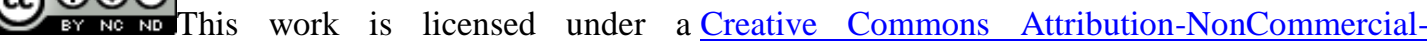
$\underline{\text { NoDerivatives } 4.0 \text { International Licens }}$

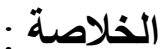

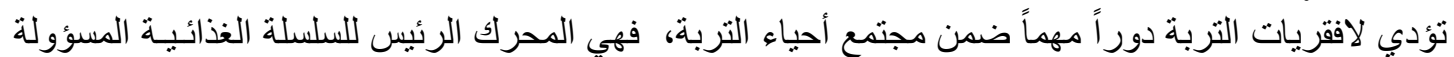

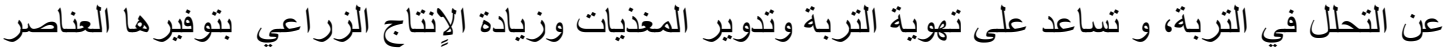

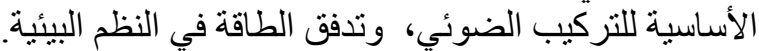

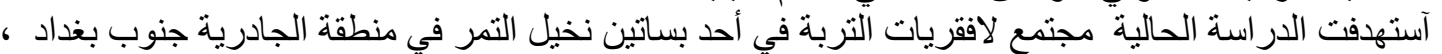

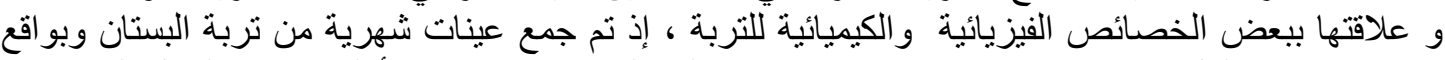

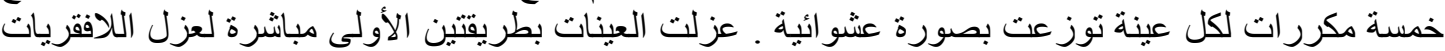

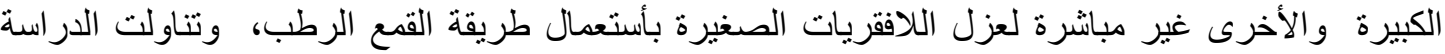

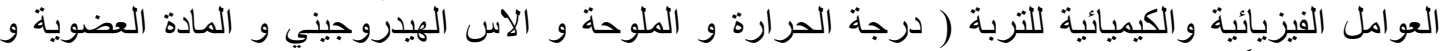

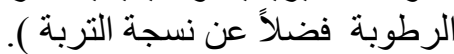

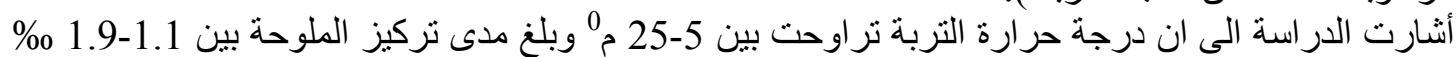

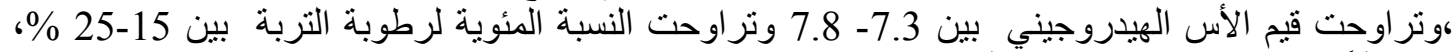

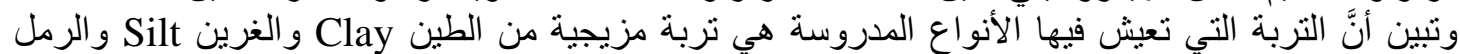

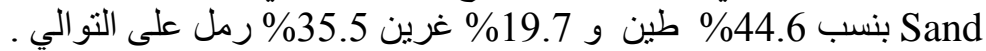

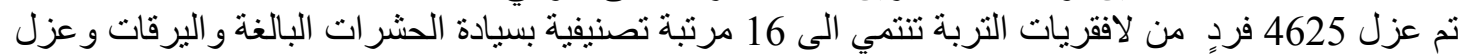

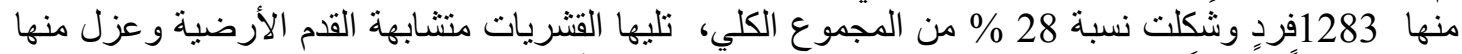

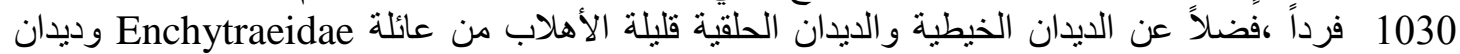

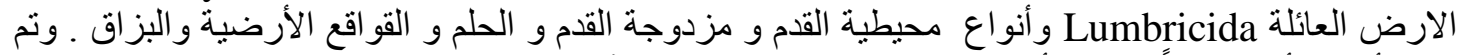
عزل أكثر الأفراد عدداً خلال الأشهر المعتدلة الحرارة (شباط واذآر ونيسان ) وبلغت 838، 813، التو الي.

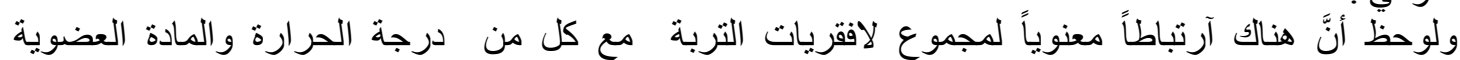
و الرطوبة. قورنت الفروق المعنوية بين المتوسطات بأستعمال آختبار أقل فرق معنوي (LSD).

الكلمات المفتاحية : محيطية القدم ، الديدان الحلقية قليلة الاهلاب ، الديدان الخيطية ، لافقريات التربة ، ديدان

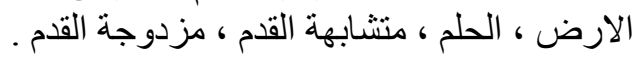

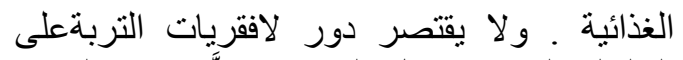

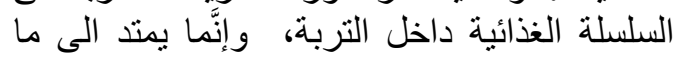

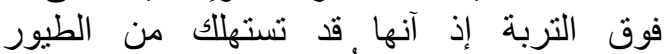

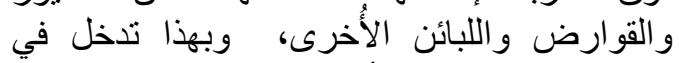

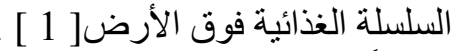
فضلاًعن دور لافقريات التربة فئل في سلسلة التحلل

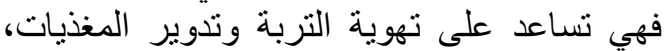

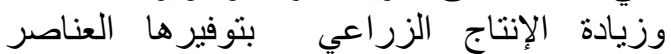

المقدمة : المقات

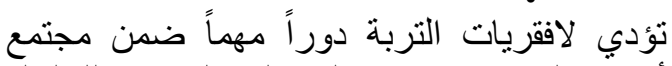

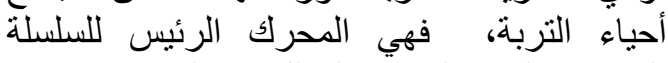

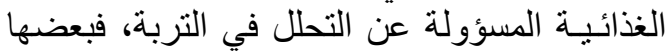

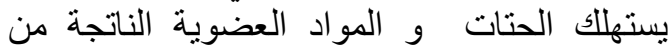
عمليات التحلل، و البعض الآخر يستهلك أكلات

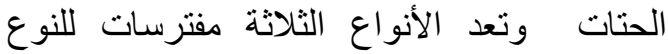

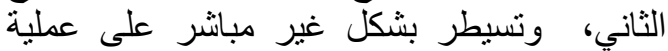
التحلل بتأثيرها في الحلقات الدنيا من السلسلة 
ظروفاً مناسبة لحصول عملية التحلل من الكائنات

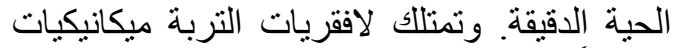

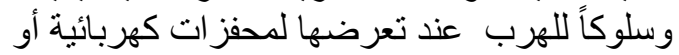

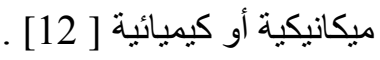

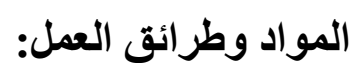

جمعت العينات بواقع العمل: خمسة مكررات شهرياً

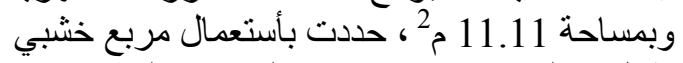

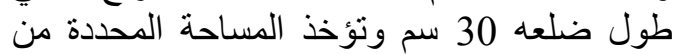

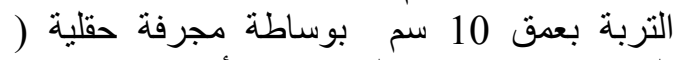
المسحاة ) ووضعت بعت العينات في أكياس بلاستيكية

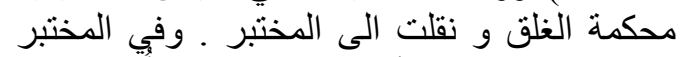

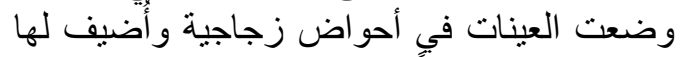

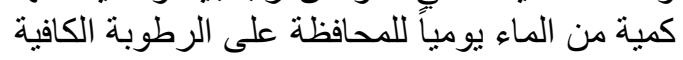
الى حين عزل اللافقريات.

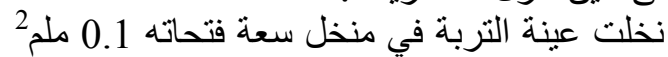

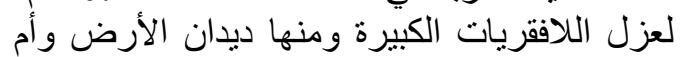

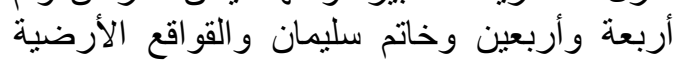

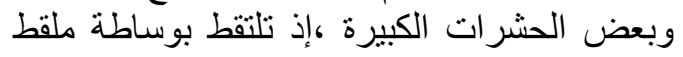

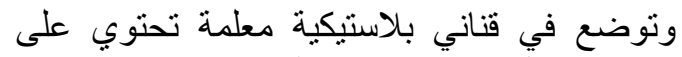

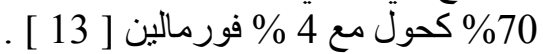
ولغرض عزل الديدان الخيطية وديدان العائلة Enchytraeidae collombola

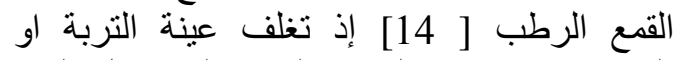

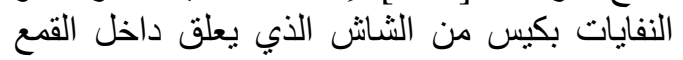

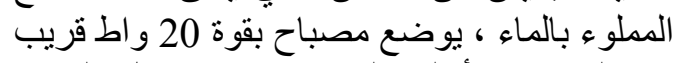

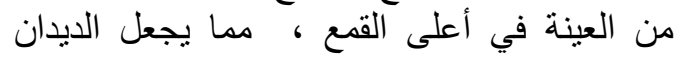

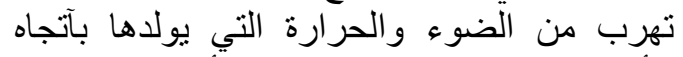

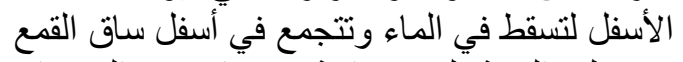

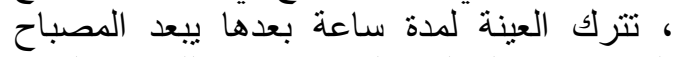

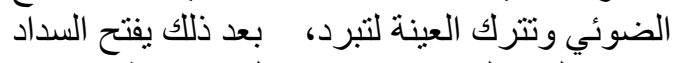

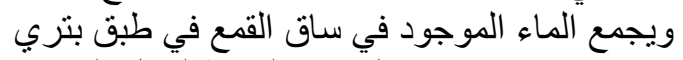
ويفحص تحت عدسة المجهر البسيط لعزل العزل الديدان.

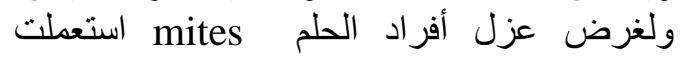
طريقة قمع تلكارن tullgren funnels method

توضع عينات الديدان الخيطية Nematoda

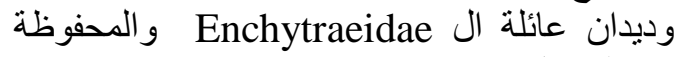

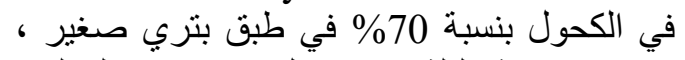

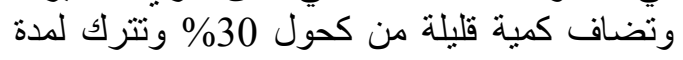

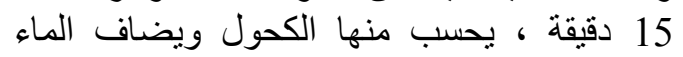

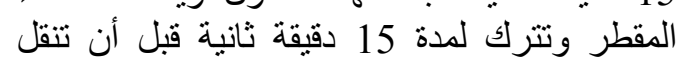

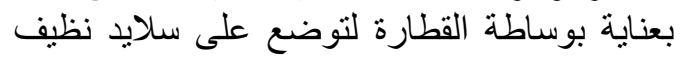

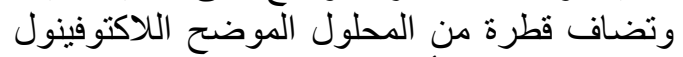
lactophenol بغطاء الشريحة Cover slip و ونترك لفئرين لمدة 24 ساعة قبل أن تفحص بوساطة المجهر المركب و Compound microscope
الأساسية للتركيب الضوئي [ 2 ] ] و تدفق الطاقة في

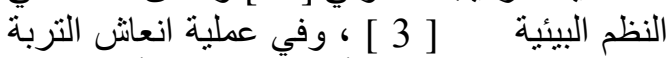

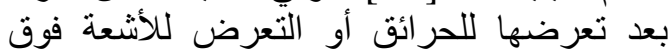
البنفسجية أو في معالجة التربة المعرضة الترضية للمبيدات

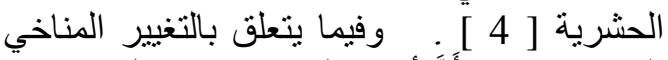

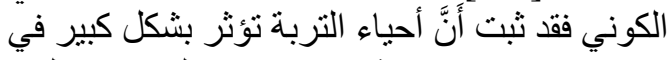

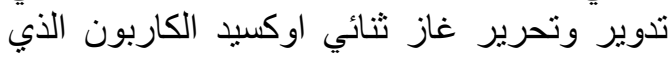

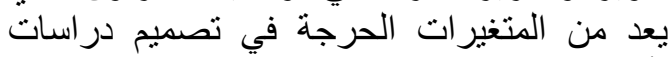
تأثير التغير المناخي [ 5 [ ] ] ل تشمل لافقريات التنربة مجاميع لافئر الافرية متنوعة

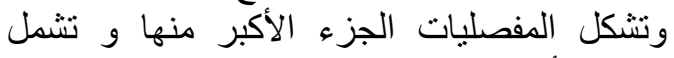
مختلف أنواع الحشرات Insecta بطوريها البالغ الغيرة

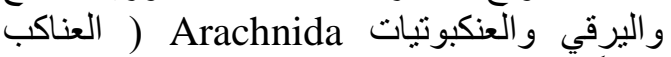

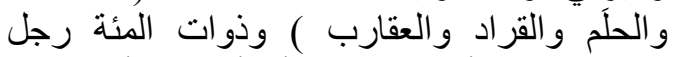

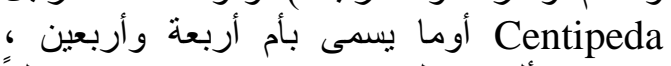

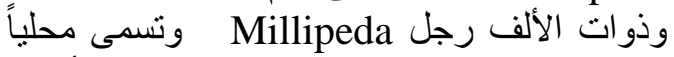
بخاتم سليمان، واتل والقتريات مزدوجة الأرجل

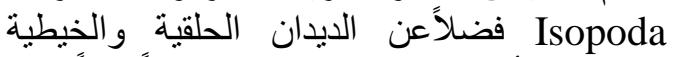

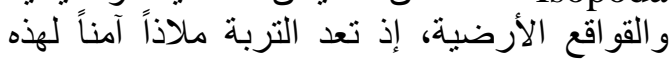

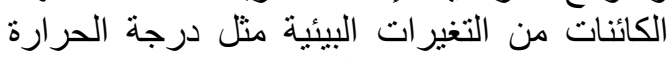

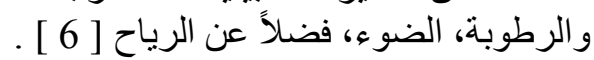

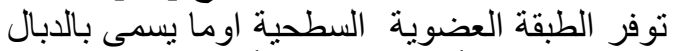

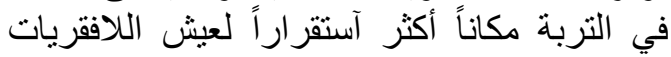

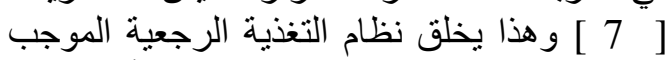
الفئ positive feedback system الغنية بالمواد العضوية تعزز وجود اللافقريات،

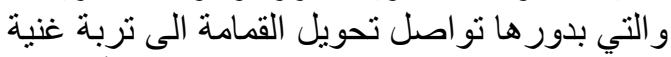

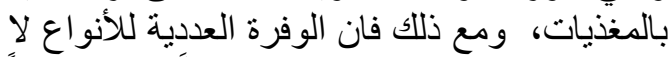

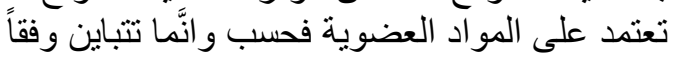

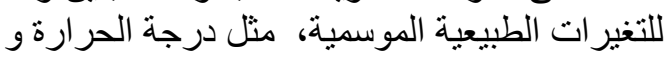

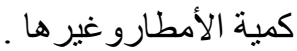
تؤثر لافقريات التربة في صحة الأبة المجتمعات النباتية

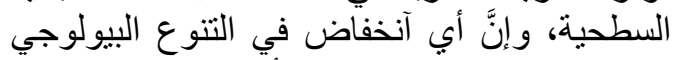

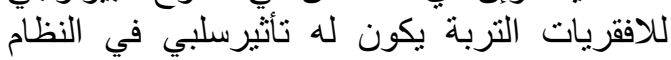

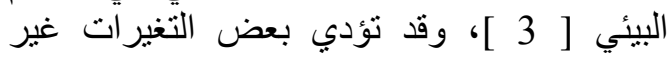

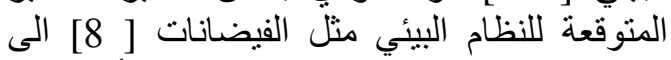

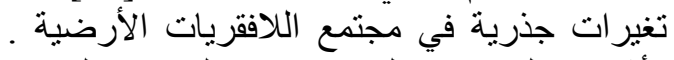

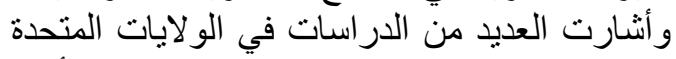

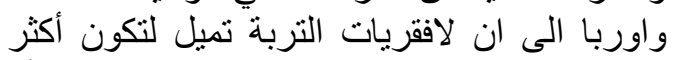

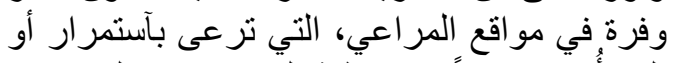

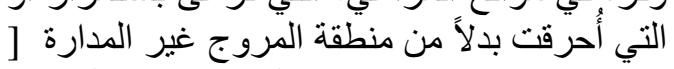

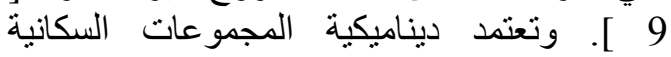

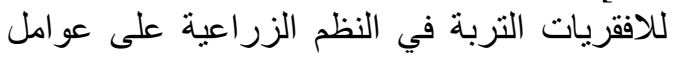

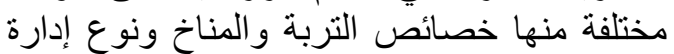

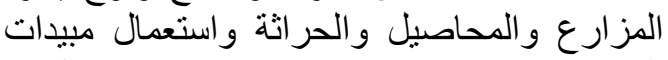

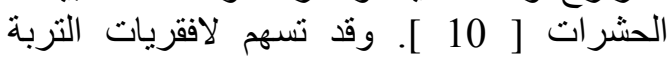

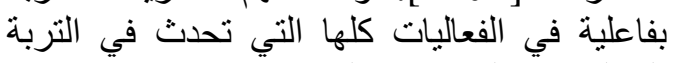

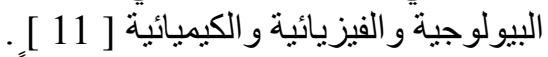

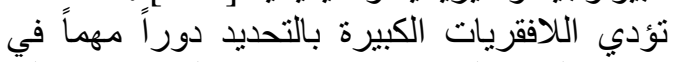
تجزئة المو اد العضوية ودمجها في التربة مما يخلق دئق دئق 
يوضح الجدول (2) عدد أفر اد اللافقريات المعزولة

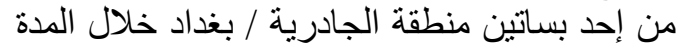

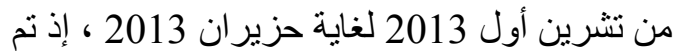
عزل 4625 فرد تتنمي الى 16 مرنبة تصنيف حنيفية بسيادة الحشرات Insecta و عزل مزل منها فرد وشكلت نسبة 28\% من مجموع الت اللافقريات

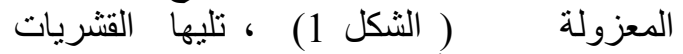

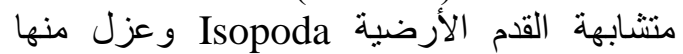
1030 فرد و القو اقع الأرضية Snails و وعزل منها ونها

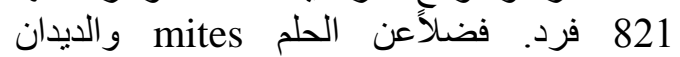
الخيطية Nematoda وقليلة الأهلاب من العائلة Enchytraeidae والعائلة Lumbricidae المعروفة بديدان الأرض اليط و والبزاق ، كنلك أم أربعة وأربعين Chilopoda وخاتم سليمان Diplopoda و أنو اع من العن العناكب. عزلت أكثر الأفراد عدداً خلال الأشهر المعتدلة العنان

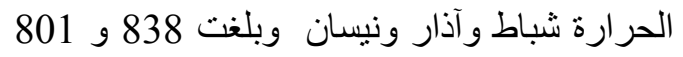

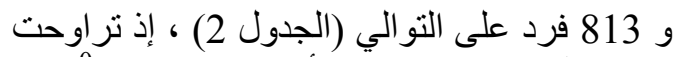

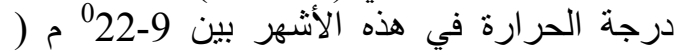

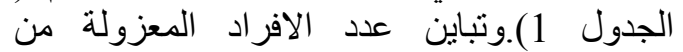

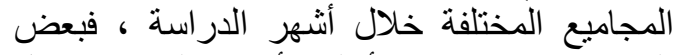

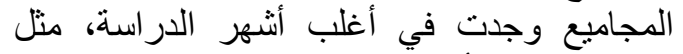
الديدان قليلة الأهلاب و القو اقع والحلم ومتشتابهة

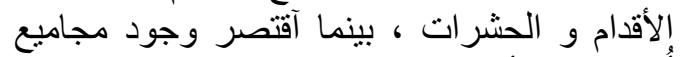

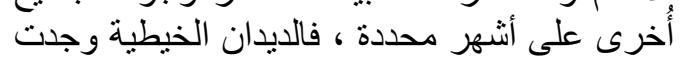
خلال الأشهر الباردة ( شُباط و آلآنار ونيسان)

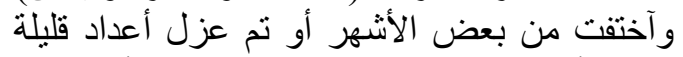

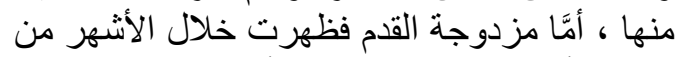
تشرين أول حتى نهاية آذار وأعلى ألى عدد لها لها عزل الانل

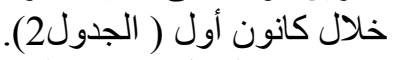

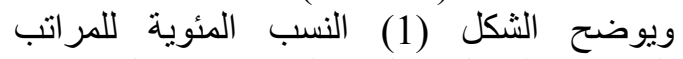
التصنيفية المختلفة والمعزولة من موقع الدراسة إذ الذية الداكية شكلت الحشرات البالغة واليرقات النئة النسبة الأكبر و التي بلغت 28\% تلتها متشابهة القدم الأرضية

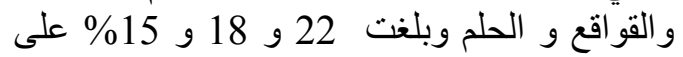

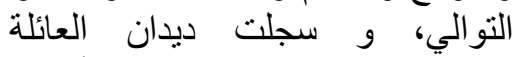
Enchytraeidae نسبة 6 \% \% أمَّا ديدان الأرض

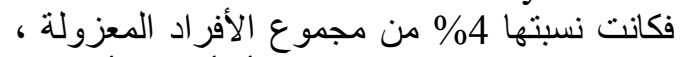
وشكلت كل من محيطية القدم ( أم أربعة وأربعين) ومزدوجة القدم ( خاتم سليمان) نسبة 2\% من الفة من

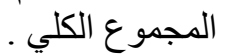
تتمايز بيئة الليابسة عموماً ومنها تربة البساتين بشكل خاص بوجود مجاميع رئيسة من ل لافقريات

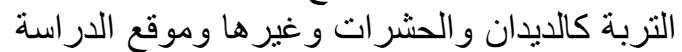

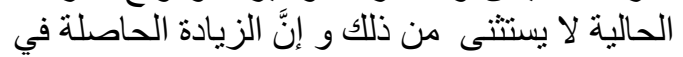

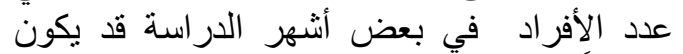

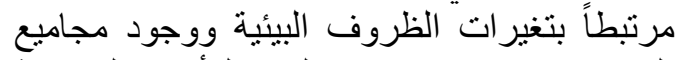
اللافقريات وتو افر مصنادر الغذاء للأنواع المفترسة . [ 16 ]
قيست أطوال اللافقريات الأرضية الكبيرة مثل الأرون

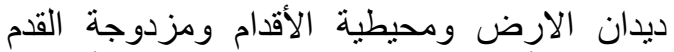
و البزاق بأستعمال المسطرة الإعتيادية ، أمَّا في الفي

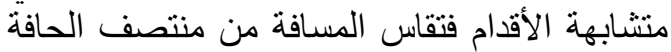

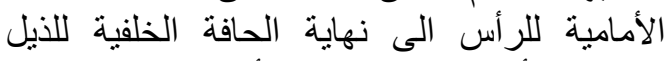
telson

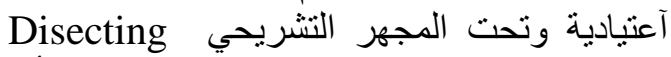
microscope الايدان الصغيرة فيتم قياس أطو الها تحت فئنة المجهر

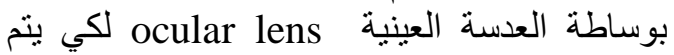
تصوير ها بوساطة العدة الصغيرة للكاميرا

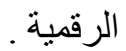

\section{النتائج و المناقشة:}

يوضح الجدول (1) قيتم المئمة العوامل الفيزياوية

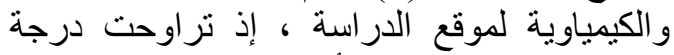

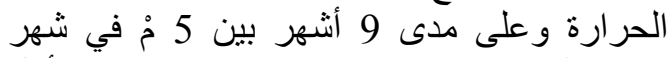

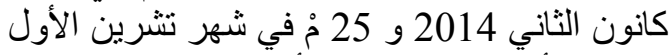

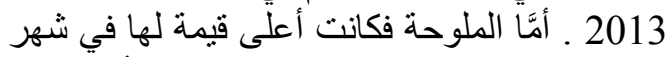
كانون الثاني وشباط ، وبلغت 1.9 \%\% و أدنى قيمة الثية لها سجلت في شهر كانون الأول 1.1 \% \%

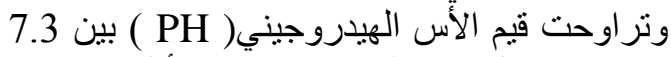

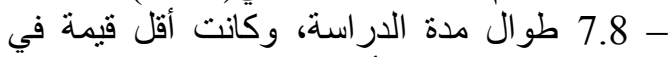

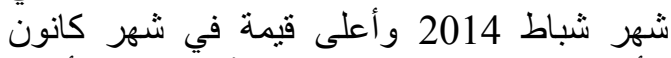
الأول 2013 ولوحظ عدم وجود آختلاف بين فين أنشهر

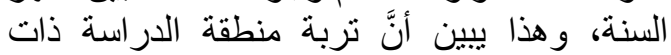

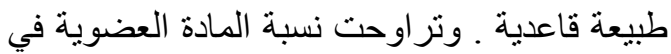
التربة خلال مدة الدراسة بين 1\% في شئ شهر تشرين

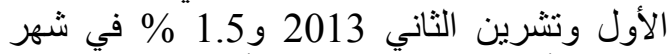
كانون الأول 2013 ـ. وسجلت أعلى قيمة لرئرئ في لرطوبة

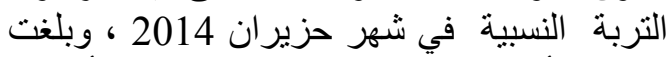

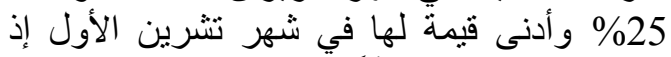

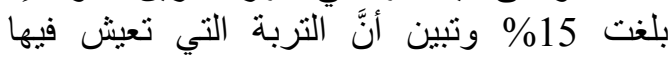

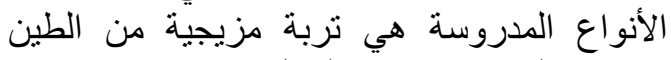

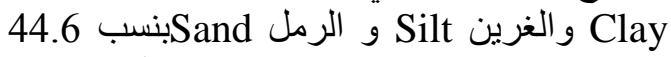
\% طين و 19.7 \% غرين 35.5 \% \% رمل .

جدول(1) : القياسات البيئية لمنطقة جمع العينات خلال المدة من تثرين الاول 2013 لغاية حزيران لينان

2014

\begin{tabular}{|c|c|c|c|c|c|}
\hline للرطوبة\% & $\begin{array}{c}\text { اللمضوية } \\
\text { \% }\end{array}$ & الهيدروجيني & الملوحة & التربة مرازة & ألشراسة \\
\hline 15 & 1 & 7.4 & 1.5 & 25 & تشثرين \\
\hline 17 & 1 & 7.5 & 1.8 & 17 & تثنرين \\
\hline 22 & 1.5 & 7.8 & 1.1 & 9 & كانول \\
\hline 20 & 1.1 & 7.5 & 1.9 & 5 & كانثاني \\
\hline 18 & 1.3 & 7.3 & 1.9 & 9 & شباط \\
\hline 18 & 1.1 & 7.4 & 1.4 & 19 & آذار \\
\hline 20 & 1.1 & 7.4 & 1.7 & 22 & نيسان \\
\hline 22 & 1.2 & 7.5 & 1.8 & 23 & أَيار \\
\hline 25 & 1.1 & 7.6 & 1.8 & 20 & حزيران \\
\hline
\end{tabular}




\begin{tabular}{|c|c|c|c|c|c|c|c|c|c|c|c|}
\hline \multirow[b]{2}{*}{$\begin{array}{l}\text { الترد } \\
\%\end{array}$} & \multirow[b]{2}{*}{$\begin{array}{l}\overline{\text { s. }} \\
\text { a }\end{array}$} & \multicolumn{9}{|c|}{ أشهر الدراسة } & \multirow[b]{2}{*}{ المرتبة التصنيفية } \\
\hline & & $\frac{1}{3}$ & 弯 & $\frac{7}{3}$ & : & 疍. & $\begin{array}{l}\frac{y}{3} \\
\frac{3}{3} \\
\overline{3}\end{array}$ & $\begin{array}{l}\frac{y}{3} \\
\frac{3}{3} \\
\overline{3}\end{array}$ & $\begin{array}{l}3 \\
3 \\
\frac{3}{3} \\
\frac{3}{3}\end{array}$ & $\begin{array}{l}3 \\
3 \\
3 \\
\frac{3}{3}\end{array}$ & \\
\hline 38 & 53 & 0 & 3 & 8 & 27 & 9 & 2 & 0 & 3 & 1 & Nematoda \\
\hline 80 & 280 & 7 & 2 & 20 & 52 & 99 & 25 & 19 & 8 & 48 & Enchytraeidae \\
\hline $\mathbf{9 8}$ & 207 & 34 & 33 & 26 & 17 & 18 & 22 & 14 & 17 & 26 & $\begin{array}{c}\text { Lumbricidae } \\
\text { (Earthworms) }\end{array}$ \\
\hline 42 & 28 & 1 & 3 & 5 & 2 & 4 & 2 & 5 & 6 & 0 & $\begin{array}{c}\begin{array}{c}\text { Arachnida - Araneae } \\
\text { (Spider) }\end{array} \\
\end{array}$ \\
\hline 76 & 689 & 15 & 23 & 225 & 106 & 201 & 47 & 52 & 20 & 0 & $\begin{array}{c}\text { Arachnida - Acarina } \\
\text { (mites) }\end{array}$ \\
\hline 100 & 1030 & 245 & 169 & 200 & 89 & 111 & 33 & 28 & 32 & 123 & $\begin{array}{c}\text { Isopoda (sow bugs, pill } \\
\text { bugs ) }\end{array}$ \\
\hline 53 & 77 & 0 & 3 & 17 & 15 & 20 & 7 & 3 & 2 & 10 & $\begin{array}{c}\text { Chilopoda } \\
\text { (Centipedes) }\end{array}$ \\
\hline 58 & 109 & 0 & 0 & 0 & 7 & 19 & 40 & 28 & 15 & 0 & Diplopoda (Millipedes) \\
\hline 100 & $\mathbf{1 2 8 3}$ & 184 & 193 & 200 & 224 & 232 & 103 & 81 & 36 & 36 & Insecta \\
\hline 84 & 821 & 22 & 54 & 105 & 256 & 123 & 130 & 101 & 30 & 0 & Gastropoda (Snails) \\
\hline 31 & 42 & 0 & 20 & 7 & 6 & 2 & 4 & 1 & 2 & 0 & Gastropoda (Slugs) \\
\hline & 4625 & 508 & 503 & 813 & 801 & 838 & 415 & 332 & 171 & 244 & المجموع - المعا \\
\hline
\end{tabular}

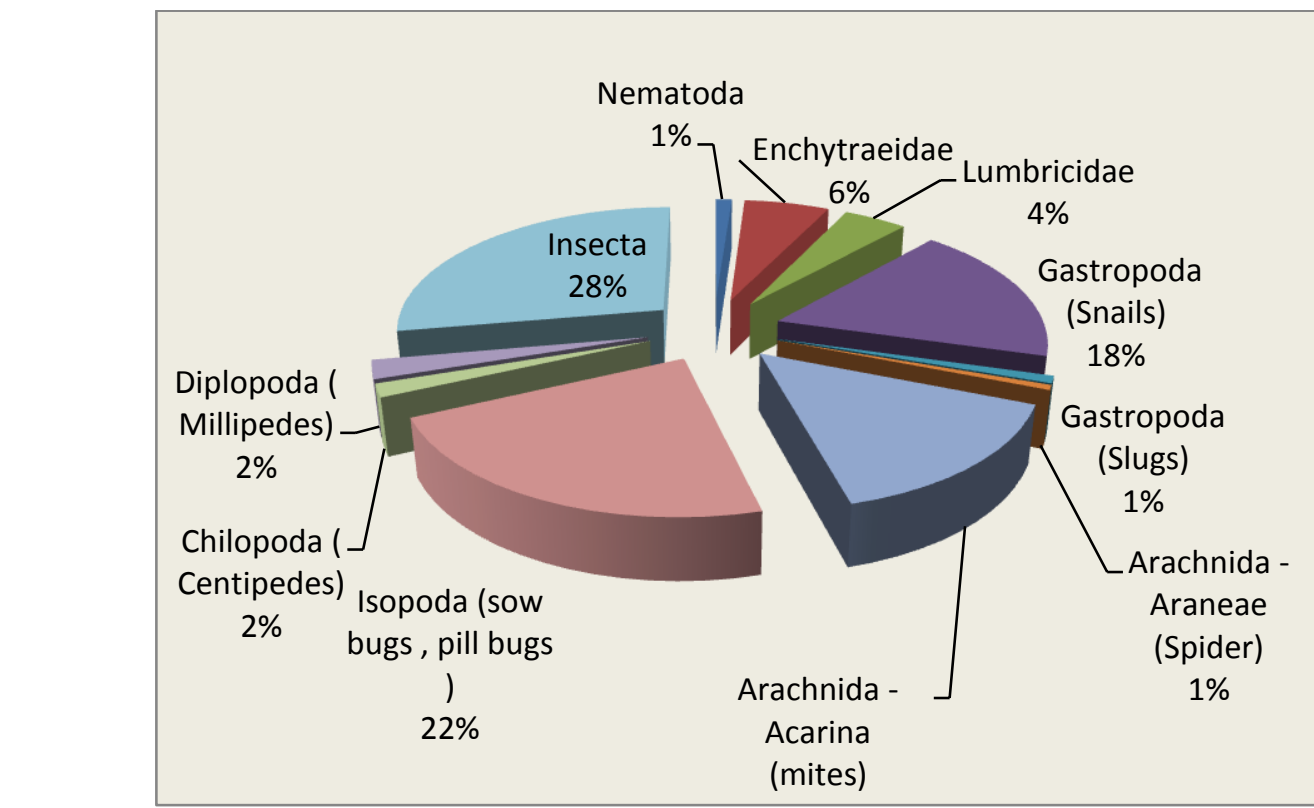

شكل (1): النسب المئوية للمجاميع المختلفة من لافقريات التربة المعزولة من إحدى البساتين في منطقة

[2] Six, J.; Feller, C.; Denef, K.; Ogle, S.M.; Moraes, J. C. and Albrecht, A. 2002. Soil organic matter, biota and aggregation in temperate and tropical Soils Effects of no-tillage .Agronomic.22:755-775.

[3] Rudd, K. 2009. Biodiversity of Soil Macroinvertebrate Communities as Influenced by Invasive Lonicera $\times$

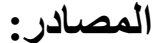

[1] Gist, C. S. and D. A. Crossely, J. r. 1975. Amodel of mineral- element cyeling for an invertebrate food web in a southeastern hard wood forest litter community. In Mineral cyeling in southeast ecosystem, pages 84106 in J. B. Gentry and M. H. Smith (eds). National Technical Information service, V. S. Dept. Commerce, Washington, DC. 
fauna under cropping system using Bt mazin. Pedobiologia, 51: 229238.

[11] Lavelle,P.; Decaens, T.; Aubert, M.; Barot, S.; Blouin, M.; Bureau, F.; Margerie, P.; Mora, P.and Rossic, J.P. 2006.Soil invertebrates and ecosystem services. Soil Biol.42:3-15.

[12] Lewbart, G.A. and Mosley, C. 2012. Clinical anesthesia and analgesia in invertebrates. J. Exo. Pet Med. 21(1):59-70.

[13] Moreno, A.G. and Mischis, C. 2004.The status of Gilberto Righis earthworm collection at the Museum of Sao Paulo. Pedobiologia, 47(24):42-46.

[14] ÓConnor, F. B. 1962. The extraction of Enchytraeide from soil in:p.w. Murphy (Ed.), Progress in Soil Zoology. Butter worths, London.

[15] Evans, G. O.; Sheals, J.G.and Macfarlane, D. 1961.The terrestrial acari of the British Isles. An introduction to their morphology, biology and classification. Trustees of the British Museum, London, P:219.

[16] AL -Qadi, B.K.H. 2011. Isolation and identification of predator soil mite species in Baghdad University Cappus in Al-Jadiriyah area with a relation to growth development of one species . B.Sc thesis. Univ. of Baghdad - Iraq. bella .BIOS569:Practicum in Field Biology.

[4] Lal, R. 2002. Soil carbon sequestration in chinathroug agricultural intensificationand relation of degraded and desertified ecosystem - land. Degardation and development. 13: 469- 478.

[5] Jikovà, V. and Frouz, J. 2014 .Contribution of ant and microbial respiration to $\mathrm{Co}_{2}$ emission from wood ant (formica polytena)nets. European. J. Soil Biol. 60:44-48.

[6] Salmon, S. and Artuso. N. 2008. Relationships between soil fauna communities and humus forms. Soil Biol. Biochem. 40:1707-1715.

[7] Chauvat, M. 2007. Humus structure during a spruce forest rotation quantitative changes and relationship to soil biota. Eur. J. Soil Biol.58(3).

[8] Tronstatd, L. M.; Tronstad, B.P. and Benk, A.C. 2005. Invertebrate seedbanks: rehydration of soil from an unregulated river flood plain in the south-eastern U.S. Freshwater Biol. 50(4): 646-655.

[9] Tscharntke, T. and Greiler, H. J. 1995. Insect communities, grasses, and grasslands. Annu. Rev. Eentomol. 40:535-558.

[10] Debeljak, M.; Coret, J.; Demsar, D.; Krogh P.H. and Dzeroski, S. 2007. Hierarchical classification of environmental factors and agricultural paratices affecting soil 


\title{
A study of Soil Invertebrates community in a Date - Palm plantation in Baghdad, Iraq
}

\author{
Haifa J. Jaweir* \\ Saba S S. Albayati** \\ *Department of Biology / College of Science for Women / University of Baghdad \\ **Ministry of Education.
}

Received 23, November, 2014

Accepted 11, January, 2015

\begin{abstract}
:
Soil invertebrates community an important role as part of essential food chain and responsible for the decomposition in the soil, helps soil aeration, nutrients recycling and increase agricultural production by providing the essential elements necessary for photosynthesis and energy flow in ecosystems.

The aim of the present study was to investigate the soil invertebrates community in one of the date palms plantation in Aljaderia district South of Baghdad, , and their relationships with some physical and chemical properties of the soil, as Five randomly distributed replicates of soil samples were collected monthly. Invertebrates samples were sorted from the soil with two methods, direct method to isolate large invertebrates and indirectly to isolate small invertebrates using wet funnel method. The study also included the determination of physical and chemical factors of the soil (Temperature, Salinity, pH, Organic matter, Humidity, In addition to the soil texture). Monthly fluctuations in physical and chemical characteristics of the soil and the total invertebrates community study site were determined. Significant correlations the of the invertebrates community and each of temperature, organic matter, and humidity were observed.

The study revealed that the temperature of the soil ranged between 5 to $25 \mathrm{C}^{0}$, The salinity concentration ranged between 1.1-1.9\%o, The $\mathrm{pH}$ values ranged between 7.3 to 7.8 and the percentage of soil moisture ranged between $15-25 \%$, Soil samples were composed of $44.6 \%$ Clay, $19.7 \%$ Silt and $35.5 \%$ Sand.

A total of 4625 individuals of soil invertebrates belonging to 16 taxa were sorted, within which the adult and larval insects were the most abundant, and from them 1283 individuals were sorted, represented $28 \%$ of the total numbers, followed by Isopoda, which 1030 individuals of them were sorted, In addition to Nematode, Oligochaetes Annelids family Enchytraeidae, and Earthworms family Lumbricida, Species of Chilopoda, Diplopoda, mites, land snails and slugs. The highest total individual number were recorded recorded durim moderate temperature months, February, March and April amounted to 838, 801 and 813 individuals, respectively.

A significant correlation was mated between total number of soil invertebrates and each of temperature, organic matter and humidity. The significant difference in means was calculated according to LSD test.
\end{abstract}

Key words: Soil invertebrate, Nematoda, Enchytraeidae , Chilopoda ,Diplopoda, Isopoda, Mites, Earthworms. 